\title{
Wie man sich bettet, so dement liegt man
}

Fordern Sie Ihren Patienten auf, sich ins Bett zu legen. Liegt er schräg, ist es höchste Zeit für einen MiniMental-Test.

— Bei über 30\% der über 60-jährigen Patienten auf internistischen Stationen ist mit einer wesentlichen kognitiven Beeinträchtigung zu rechnen, die in der klinischen Routine leider oft unentdeckt bleibt. Neurologen aus Würzburg haben nun eine einfache Situation aus dem klinischen Alltag bezüglich ihrer Eignung als Demenztest validiert.

110 Patienten einer Neurologischen Station im medianen Alter von knapp 71 Jahren wurden aufgefordert, sich ins Bett zu legen. Mittels einer verborgenen Digitalkamera wurde ein Foto des Bettes und des Patienten gemacht. Sämtliche Patienten wurden mit der Mini-Mental State Examination (MMSE), dem DemTect-Test und dem Uhren-Test auf eine mögliche kognitive Beeinträchtigung hin untersucht.
Ein Untersucher, der keine Kenntnis vom Ergebnis der Tests hatte, bestimmte anhand der Fotos den Winkel zwischen der Längsachse des Bettes und der Körperlängsachse des Patienten (siehe Abb.). Der absolute Wert dieses Winkels reichte von 0-23 Grad mit einem Median von 3 Grad. $24 \mathrm{~Pa}-$ tienten wiesen Hinweise auf eine kognitive Beeinträchtigung auf, acht hatten nach den Definitionskriterien des MMSE und elf anhand des DemTectTests eine Demenz.

Zwischen der Größe des Winkels und dem Ergebnis der kognitiven Tests bestand eine eindeutige Korrelation, die auch nach Korrektur für das Alter bestehen bleib.

\section{Kommentar}

Es könnte durchaus sein, dass Sie das Phänomen einer schiefen Lage im Bett schon öfter mehr oder weniger bewusst registriert haben, ohne sich über dessen Bedeutung im Klaren zu sein. Wieder ein
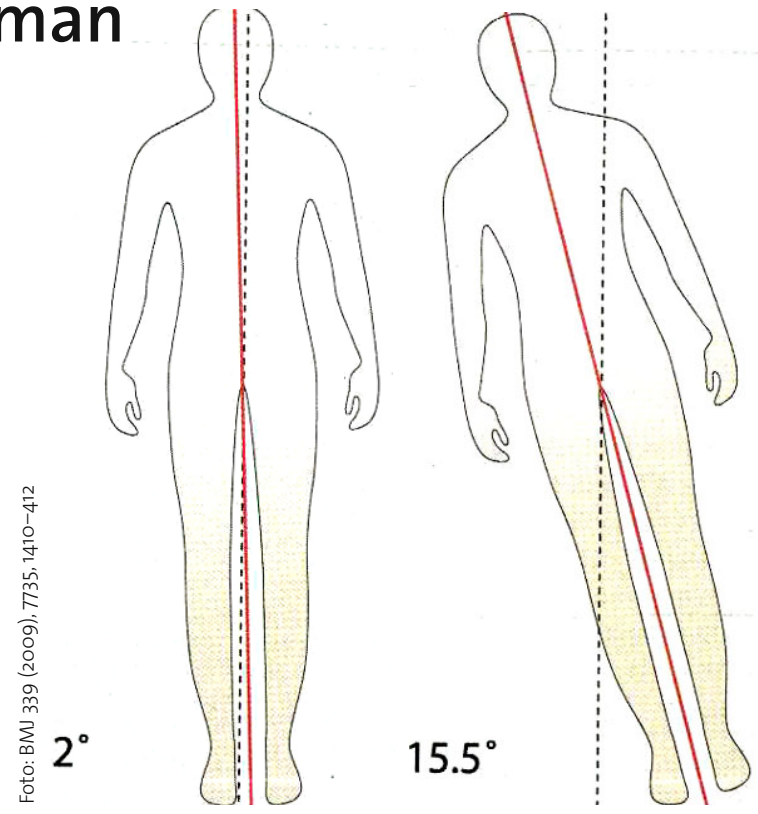

"Gesunder" und „dementer“ Winkel im Vergleich.

Beispiel dafür, wie Wissenschaft häufig mit einfachsten Beobachtungen beginnt.

- P. Kraft et al.

H. S. FüEßL =

Lying obliquely - a clinical sign of cognitive impairment: cross sectional observational study. BMJ 339 (2009), 7735, 1410-412

\section{Neurologie - der ideale Stoff für Seifenopern}

- Neurologische Krankheiten scheinen in Kasuistiken medizinischer Fachzeitschriften, aber auch in Ärzteserien im Fernsehen überproportional häufig vorzukommen. Ob das tatsächlich stimmt, und welche Gründe das haben könnte wurde nun von zwei englischen Wissenschaftlern anhand zweier wichtiger Medizinjournale und der Ärzteserie „Dr. House“ untersucht.

Dr. Gregory House, der Star der amerikanischen Ärzteseifenoper „House MD“, ist ein zum Zynismus neigender, misanthropischer und einzelgängerischer Arzt, der in der Art von Sherlock Holmes diagnostisch verzwickte Fälle aufklärt. Unter den von Dr. House diagnostizierten Fällen liegen neurologische Krankheiten mit 27,5\% aller Fälle mit weitem Abstand an erster Stelle, gefolgt von Infektionskrankheiten mit 16\% und genetischen bzw. hämatologischen Krankheiten mit jeweils 6,5\%.

Auch bei den Fallberichten des British Medical Journal liegt die Neurologie mit 17,3\% an der Spitze der Hitliste, an zweiter Stelle stehen kardiologische Fälle mit 16,7\% und an dritter Stelle onkologische Krankheiten mit $8,7 \%$.

Diese Hitliste steht im krassen Gegensatz zur tatsächlichen Epidemiologie. In der realen Welt liegen psychiatrische Krankheiten mit 22,3\% an der Spitze, gefolgt von kardiologischen mit $14,5 \%$ und onkologischen mit $14,2 \%$. Eine ähnlich schiefe Verteilung zugunsten der Neurologie findet sich auch im Lancet.

\section{Kommentar}

Die Vielzahl oft bizarrer Symptome bei neurologischen Erkrankungen eignet sich gut für einen unterhaltsamen Fallbericht. Das große Interesse an eigenartigen neurologischen Fällen zeigt sich auch am Publikumserfolg des Neurologen Oliver Sacks (,Der Mann, der seine Frau für einen Hut hielt"). Es ist schwer vorstellbar, dass ein Buch mit dem Titel „Der Mann, der häufig blutige Stühle absetzte" sich zu einem ähnlichen Renner entwickeln könnte. Neurologische Krankheiten haben alle Zutaten eines guten Dramas. Kein Wunder, dass sich auch Ärzteserien im Fernsehen darauf stürzen.

H. S. FÜEßL .

- R. H. Thomas, N. J. Thomas

(rhys-thomas@doctors.org.uk). House calls. BMJ 339 (2009), 7735, 1416-1417. 